\title{
Clustering and Halo Occupation Distribution of Active Galactic Nuclei
}

\author{
Takamitsu Miyaji, M. Krumpe, A. Coil, H. Aceves and B. Husemann \\ Inst. de Astron. sede Ensenada, Univ. Nacional Autonoma de Mexico \\ and UCSD CASS, Mexico/USA \\ E-mail: miyaji@astrosen.unam.mx
}

\begin{abstract}
We present the results of our series of studies on correlation function and halo occupation distribution of AGNs utilizing data the ROSAT All-Sky Survey (RASS) and the Sloan Digital Sky Survey (SDSS) in the redshift range of $0.07<\mathrm{z}<0.36$. In order to improve the signal-to-noise ratio, we take cross-correlation approach, where cross-correlation functions (CCF) between AGNs and much more numerous AGNs are analyzed. The calculated CCFs are analyzed using the Halo Occupation Distribution (HOD) model, where the CCFs are divided into the term contributed by the AGN-galaxy pairs that reside in one dark matter halo (DMH), (the 1-halo term) and those from two different DMHs (the 2-halo term). The 2-halo term is the indicator of the bias parameter, which is a function of the typical mass of the DMHs in which AGNs reside. The combination of the 1-halo and 2-halo terms gives, not only the typical DMH mass, but also how the AGNs are distributed among the DMHs as a function of mass separately for those at the center of the DMHs and satellites. The main results are as follows: (1) the range of typical mass of the DMHs in various sub-samples of AGNs $\log \left(\mathrm{M}_{D M H} / \mathrm{h}^{-1} \mathrm{M}_{\Theta}\right) \sim 12.4-13.4$, (2) we found a dependence of the AGN bias parameter on the X-ray luminosity of AGNs, while the optical luminosity dependence is not significant probably due to smaller dynamic range in luminosity for the optically-selected sample, and (3) the growth of the number of AGNs per DMH $\left(\mathrm{N}\left(\mathrm{M}_{D M H}\right)\right)$ with $\mathrm{M}_{D M H}$ is shallow, or even may be flat, contrary to that of the galaxy population in general, which grows with $\mathrm{M}_{D M H}$ proportionally, suggesting a suppression of AGN triggering in denser environment. In order to investigate the origin of the X-ray luminosity dependence, we are also investigating the dependence of clustering on the black hole mass and the Eddington ratio, we also present the results of this investigation.
\end{abstract}

